\title{
ZigBee Ranging using Phase Shift Measurements
}

\author{
Jacek Rapinski ${ }^{1}$ and Michal Smieja ${ }^{2}$ \\ ${ }^{I}$ (Institute of Geodesy, University of Warmia and Mazury in Olsztyn, Poland) \\ ${ }^{2}$ (Chair of Mechatronics, University of Warmia and Mazury in Olsztyn, Poland) \\ (E-mail: jacek.rapinski@uwm.edu.pl)
}

\begin{abstract}
This paper presents the results of distance measurements performed with an AT86RF233 chip. It uses a combination of time of flight and phase shift measurements to perform ranging. The statistical parameters describing the ranging results are presented and an algorithm to process raw measurement data is proposed. The results show significant improvement in ranging accuracy.
\end{abstract}

\section{KEY WORDS}

1. Ranging. 2. ZigBee. 3. Phase shift measurement.

Submitted: 15 July 2014. Accepted: 2 January 2015. First published online: 26 January 2015.

1. INTRODUCTION. In the field of navigation and positioning, technologies based on Radio Frequency (RF) wave propagation are gaining increasing attention. There are various positioning techniques and systems - from global (operating worldwide) to local or personal navigation (operating only inside a particular area, certain place or application). In recent years, the use of local area RF positioning has gained attention, especially in applications where Global Navigation Satellite Systems (GNSS) techniques are not usable (e.g. indoor positioning). RF communication technology is regulated by the IEEE 802.15.4 standard. The use of standardised communication technologies makes positioning much more effective, especially in positioning systems comprised of more than one location device. Since, from a geometric point of view, positioning in RF networks is very often based on trilateration, position accuracy depends on the distance measurement accuracy. There are several different approaches to obtain the distance between nodes in RF technology, including the utilisation of Radio Signal Strength (RSS) (Chen et al., 2012; Wu et al., 2009) to techniques involving Time Of Flight (TOF), Time Of Arrival (TOA), or angle of arrival associated with their communication functionality (Günther and Hoene, 2005; Indelman et al., 2011; Nilson et al., 2013; Obst et al., 2012). Though these techniques show some promising results, the accuracy of such positioning can be improved to allow its use in more demanding applications (Smieja, 2014). Improvement in positioning accuracy can be obtained either by developing new positioning or ranging algorithms, or by new measurement techniques. 


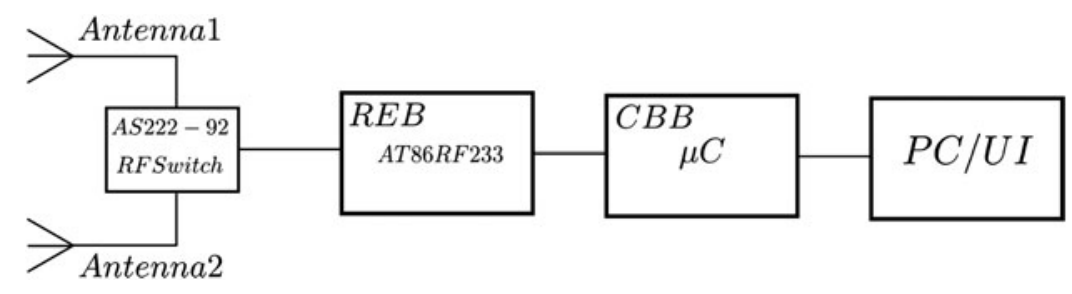

Figure 1. Device block diagram.

A new ranging approach based on the integration of ZigBee communication network protocol and phase shift measurements of RF waves is implemented in the AT86RF233 chip, which provides the possibility to measure distance with much better accuracy than previous techniques. The AT86RF233 is equipped with a Phase Measurement Unit (PMU), which makes it possible to perform ranging using a phase shift measurement as well as perform TOF measurements.

The key advantage of the ZigBee device is its low power consumption. A single device can operate for a very long time (up to several years) on a single battery. It can be used for small mobile devices or embedded into modern cell phones or tablets. A detailed description of ZigBee power consumption can be found in Casilari et al. (2010).

This paper presents the results of preliminary ranging tests using phase difference measurements based on ZigBee. In this paper, the AT86RF233 chip-ranging results are investigated, and an algorithm to process the measurement results is proposed.

2. DEVICE DESCRIPTION. In the experiment a REB233SMAD-EK device was used. It consists of a pair of network nodes, called "initiator" and "reflector" - as illustrated in Figure 3. Each of the individual nodes consists of a Radio Extender Board (REB) equipped with RF233 AT86RF233 and a Controller Base Board (CBB) equipped with ATxmega256a3 micro-controller (Atmel, 2013). As the user interface a Personal Computer (PC) is used, connected with the initiator node using a Universal Serial Bus (USB) line. The block diagram of the device is presented in Figure 1.

An important feature of an AT86RF233 Transceiver, dedicated primarily for communication in accordance with protocols such as ZigBee, RF4CE, I 6LoWPAN, is the ability to perform ranging, thanks to embedded peripherals such as a Phase Measurement Unit (PMU) or a Time of Flight Module (TOM). In ranging mode, the AT86RF233 chip operates in the $2 \cdot 4 \mathrm{GHz}$ RF band. The power consumption of the AT86RF233 is approximately $1 \mu \mathrm{A}$ in sleep state and up to $14 \mathrm{~mA}$ in active transmit state. Since one of the major disturbances in such measurements is the multipath effect caused by the impact of the environment, to decrease the impact of this effect there is an AS222-92 RF-switch and two pole antennas in the input antenna circuit, as shown in Figure 1. The REB233SMAD-EK device is programmed to perform the ranging procedure and to communicate between the nodes according to the rules based on IEEE802 $15 \cdot 4$.

The ranging process between two nodes, preceded by a synchronization phase, is based on the measurement of the RF signal phase shift between the nodes. During 
NO. 4

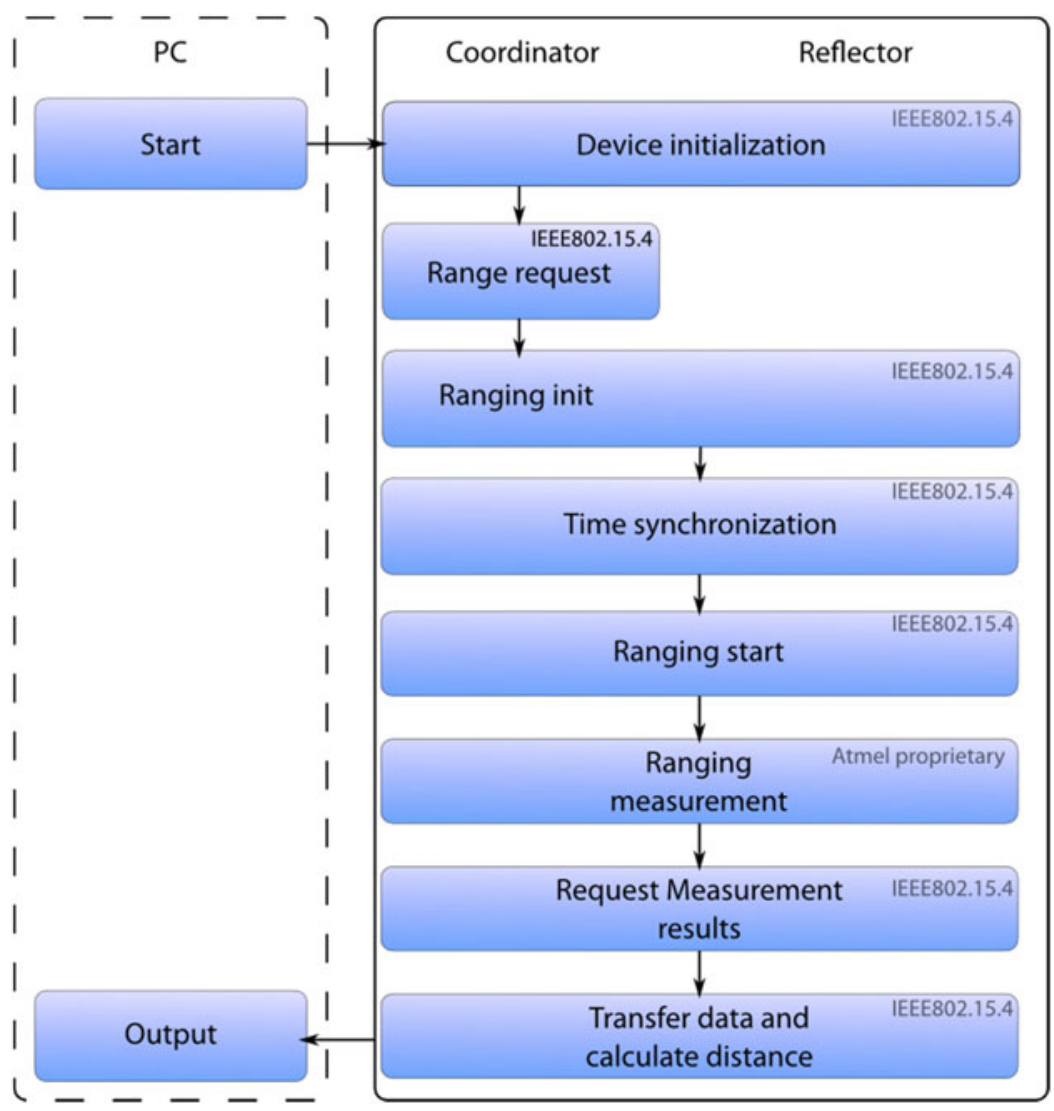

Figure 2. Ranging procedure (Atmel, 2013).

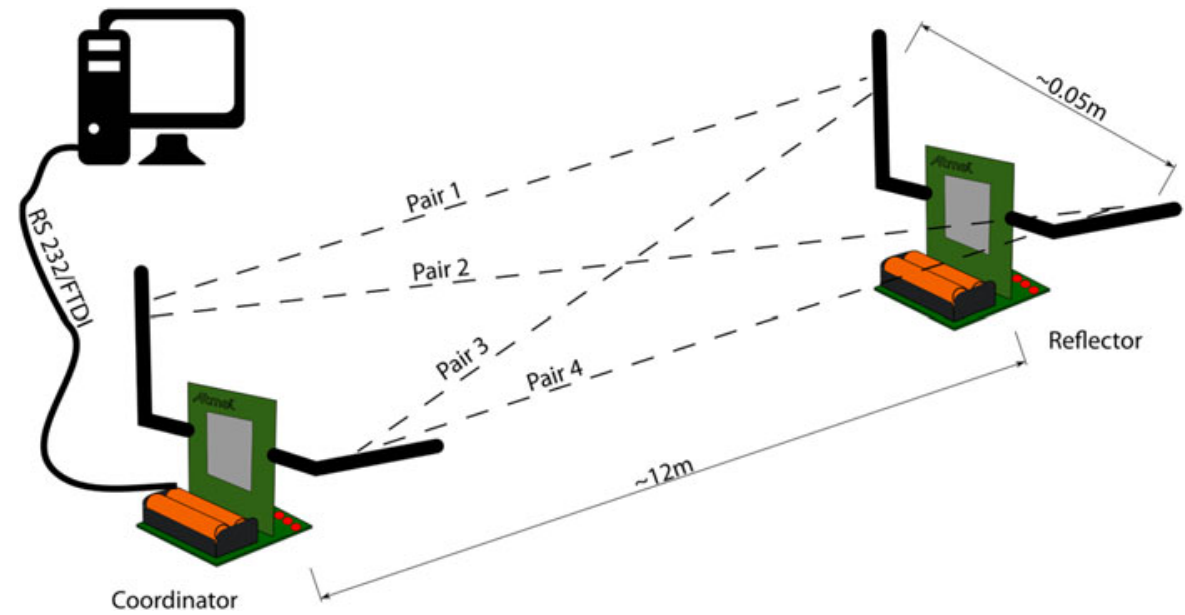

Figure 3. Device configuration. 


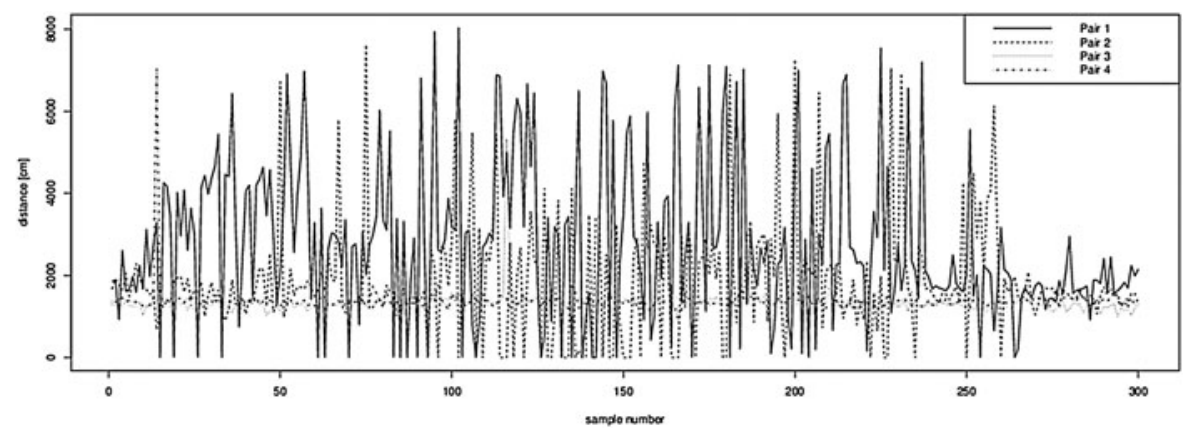

Figure 4. Raw measurement results.

one ranging cycle the action is repeated several times for an assumed number of selected frequencies. Every single result is complemented with an additional parameter $v$ that reflects the correctness of the measurement $(v=1$ is a valid result and $v=0$ is invalid result). The final result of one cycle is expressed as a pair [d; DQF] where $d$ is the calculated and averaged distance and DQF is a Distance Quality Factor (\% of valid results in a ranging cycle). The exchange of the frames carrying necessary information such as a request or answer or confirmation, is based on IEEE802.15.4 excluding the actual ranging phase. This is REB233SMAD-EK specific, as depicted in Figure 2.

3. EXPERIMENT. The test measurements were conducted using two nodes placed on known reference baselines, as depicted in Figure 3.

The location of the nodes during each experiment was constant and the reference distances between the nodes were $1=4.86 \mathrm{~m}, 1=12.76 \mathrm{~m}, 1=20.00 \mathrm{~m}, 1=43.80 \mathrm{~m}$, $1=62 \cdot 00 \mathrm{~m}$. The reference distances were measured with a laser rangefinder. The distance between the antennas mounted on a single node and working in the diversity mode was $12 \mathrm{~cm}$. In further considerations it was assumed to be 0 . This assumption is justified because the distance between nodes is from a few metres up to 40-50 metres, while the distance between antennas in a single device is about $10 \mathrm{~cm}$. Therefore, four measured distances are considered as the same distance with an expected accuracy of $10 \mathrm{~cm}$. The vertical location of both nodes was $h=1.50 \mathrm{~m}$ above the ground level. Between the nodes there were no obstacles in the Line Of Sight (LOS) or in the close vicinity.

The experiment was performed in two stages. First a series of 300 measurements of distances between nodes 1 and 2 was performed with a $100 \mathrm{~ms}$ time interval at each distance. The results from four pairs of antennas (as shown as an example in Figure 4 for $12.76 \mathrm{~m}$ ) were stored in a file, including distances with corresponding DQF factors.

In the second stage, the results were processed with the algorithm described in Section 4 and were then analysed.

4. EVALUATION OF THE RESULTS AND ALGORITHM FOR DISTANCE DATA PROCESSING. As an example, results of the obtained distance measurements at $12.76 \mathrm{~m}$ (which will be called raw measurements in the paper) are depicted 
Table 1. Statistics of the raw distance measurement $[\mathrm{cm}]$.

\begin{tabular}{|c|c|c|c|c|c|c|}
\hline Pair & $\operatorname{Min}[\mathrm{cm}]$ & Median $[\mathrm{cm}]$ & Mean $[\mathrm{cm}]$ & $\operatorname{Max}[\mathrm{cm}]$ & $\sigma[\mathrm{cm}]$ & $\delta[\%]$ \\
\hline \multicolumn{7}{|c|}{$486 \mathrm{~cm}$} \\
\hline 1 & 369 & 493 & 492 & 542 & 18 & $1 \cdot 23$ \\
\hline 2 & 400 & 476 & 476 & 533 & 20 & $2 \cdot 05$ \\
\hline 3 & 312 & 469 & 468 & 586 & 39 & $3 \cdot 70$ \\
\hline 4 & 266 & 502 & 498 & 564 & 25 & $2 \cdot 46$ \\
\hline \multicolumn{7}{|c|}{$1276 \mathrm{~cm}$} \\
\hline 1 & 0 & 2450 & 2769 & 8041 & 1906 & $117 \cdot 01$ \\
\hline 2 & 0 & 1632 & 1843 & 7627 & 1370 & $44 \cdot 43$ \\
\hline 3 & 991 & 1328 & 1337 & 5330 & 255 & $4 \cdot 78$ \\
\hline 4 & 892 & 1387 & 1387 & 7030 & 344 & $8 \cdot 70$ \\
\hline \multicolumn{7}{|c|}{$2000 \mathrm{~cm}$} \\
\hline 1 & 2178 & 2463 & 2458 & 2761 & 97 & $22 \cdot 90$ \\
\hline 2 & 1753 & 2242 & 2226 & 2324 & 156 & $11 \cdot 3$ \\
\hline 3 & 1560 & 2129 & 2126 & 2453 & 149 & $6 \cdot 3$ \\
\hline 4 & 1794 & 2487 & 2437 & 2764 & 169 & $21 \cdot 85$ \\
\hline \multicolumn{7}{|c|}{$4380 \mathrm{~cm}$} \\
\hline 1 & 0 & 4164 & 4177 & 7096 & 891 & $4 \cdot 63$ \\
\hline 2 & 2926 & 4377 & 4356 & 5348 & 213 & $0 \cdot 55$ \\
\hline 3 & 0 & 4321 & 4072 & 7123 & 1462 & $7 \cdot 03$ \\
\hline 4 & 3943 & 4472 & 4458 & 4708 & 114 & $1 \cdot 78$ \\
\hline \multicolumn{7}{|c|}{$6200 \mathrm{~cm}$} \\
\hline 1 & 0 & 6213 & 6015 & 7129 & 917 & 2.98 \\
\hline 2 & 0 & 6578 & 6152 & 7135 & 1596 & 0.77 \\
\hline 3 & 0 & 6228 & 6170 & 7149 & 537 & $0 \cdot 48$ \\
\hline 4 & 0 & 6226 & 5974 & 6996 & 1078 & $3 \cdot 64$ \\
\hline
\end{tabular}

in Figure 4. The distance measurement statistics for all measured distances are presented in Table 1. The relative error $\delta$ is defined as:

$$
\delta=\frac{\left|\bar{d}-d_{0}\right|}{d_{0}} 100 \%
$$

where $\bar{d}$ is the average of all samples from one measurement and $d_{0}$ is the reference distance.

Explicit differences between distances from each pair are caused mainly by a multipath effect, which is typical in such kinds of ranging technology (Liu et al., 2007). In the experiment, the multipath effect is caused by RF waves reflected from the floor. As a result of this phenomenon, fading occurs which causes interference in measurement results. The presence of fading due to interference in LOS causes an unpredictable effect of constructive or destructive interference in RF signal reception that is difficult to eliminate. This disturbance depends strongly on the location of the transmitting and receiving antenna.

The minimum, maximum and standard deviations presented in Table 1 reflect this phenomenon and indicate which pair of antennae is not affected by multipath. The 
Pair 1

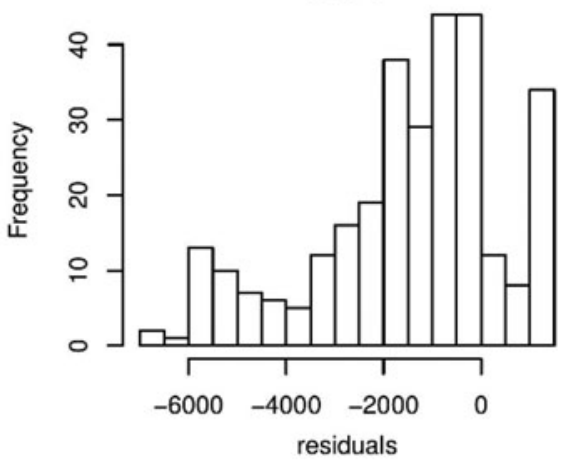

Pair 3

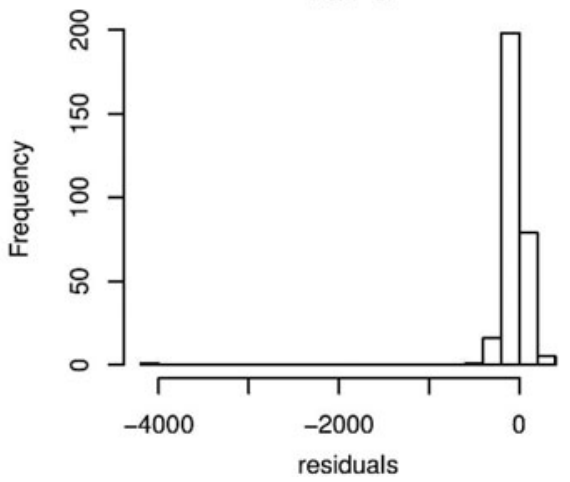

Pair 2

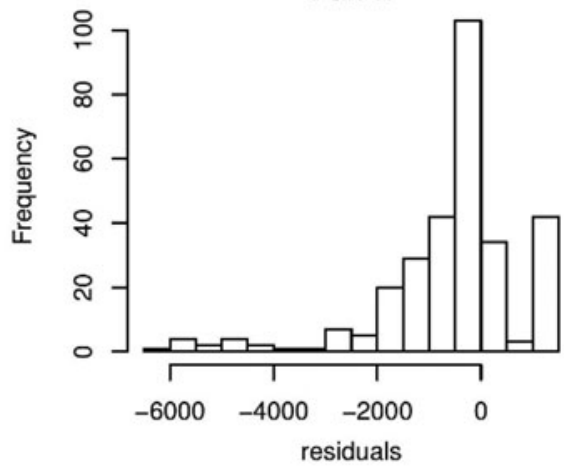

Pair 4

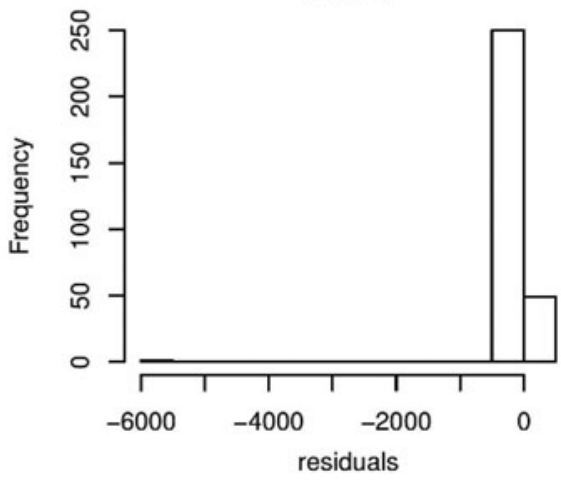

Figure 5. Histograms of calculated distance residuals for $12 \cdot 76 \mathrm{~m}$.

antenna diversity applied in the device allows it to choose the data from the less disturbed pair of antennae, thanks to their slightly different locations.

At each distance the relative error and standard deviations vary depending on the antenna pair. For example at the mean distance of $12.76 \mathrm{~m}$, distances (calculated from all cycles) are in the boundary of $1 \sigma_{t}$ for pairs 3 and 4 , while the values from pairs 1 and 2 differ significantly. This is also confirmed by the histograms in Figures 5 and 6.

Figure 5 shows the histogram of residuals for all antenna pairs. The residuals were calculated using Equation (2).

$$
v_{i}=D-d_{i}
$$

where $v_{i}$ is the residual, $D$ is the reference distance and $d_{i}$ is the measurement result for one pair of antennae.

At $12.76 \mathrm{~m}$, correct distances (within $1 \sigma_{t}$ boundary) are present for each pair, although pairs 3 and 4 have the highest number of correct results. This is confirmed by the DQF values (Figure 6). In the case of pairs 3 and 4 there is a much higher number of DQFs with a high value than for pairs 1 and 2 .

Another important value for signals is their distribution. As an example, a comparison of the distribution of $12.76 \mathrm{~m}$ distance measurements for each pair of antennae 
Pair 1

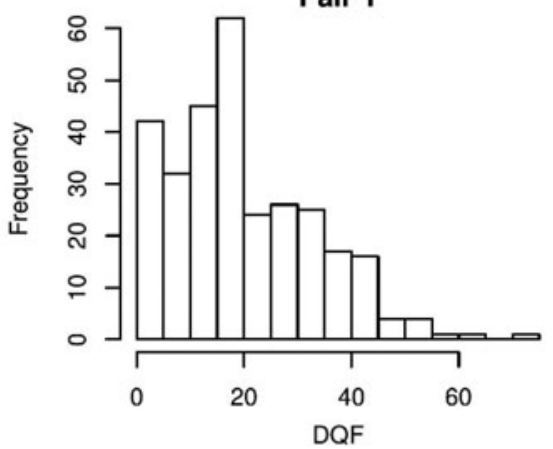

Pair 3

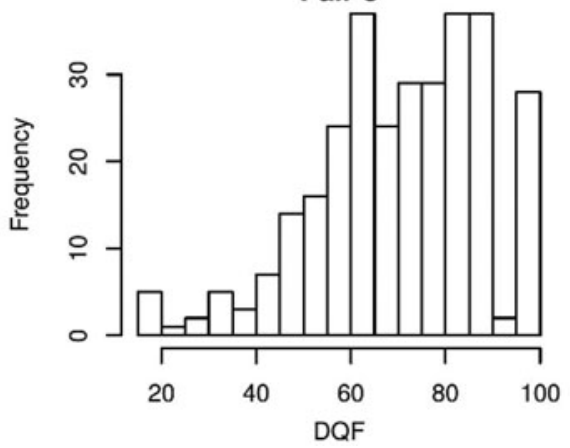

Pair 2

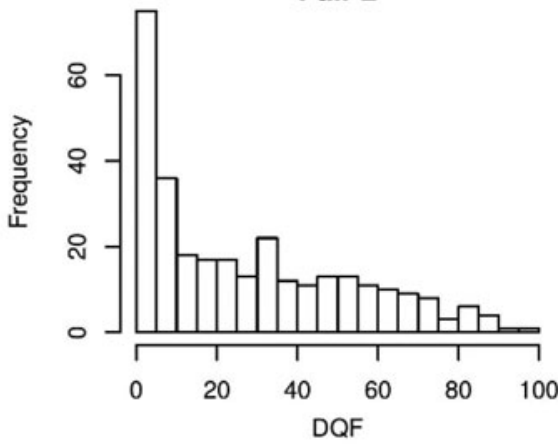

Pair 4

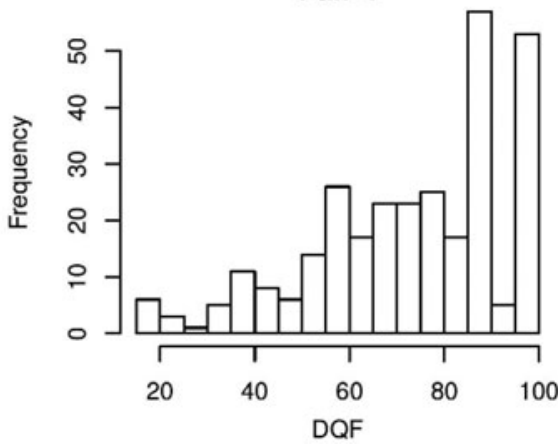

Figure 6. Histograms of DQF for $12 \cdot 76 \mathrm{~m}$.
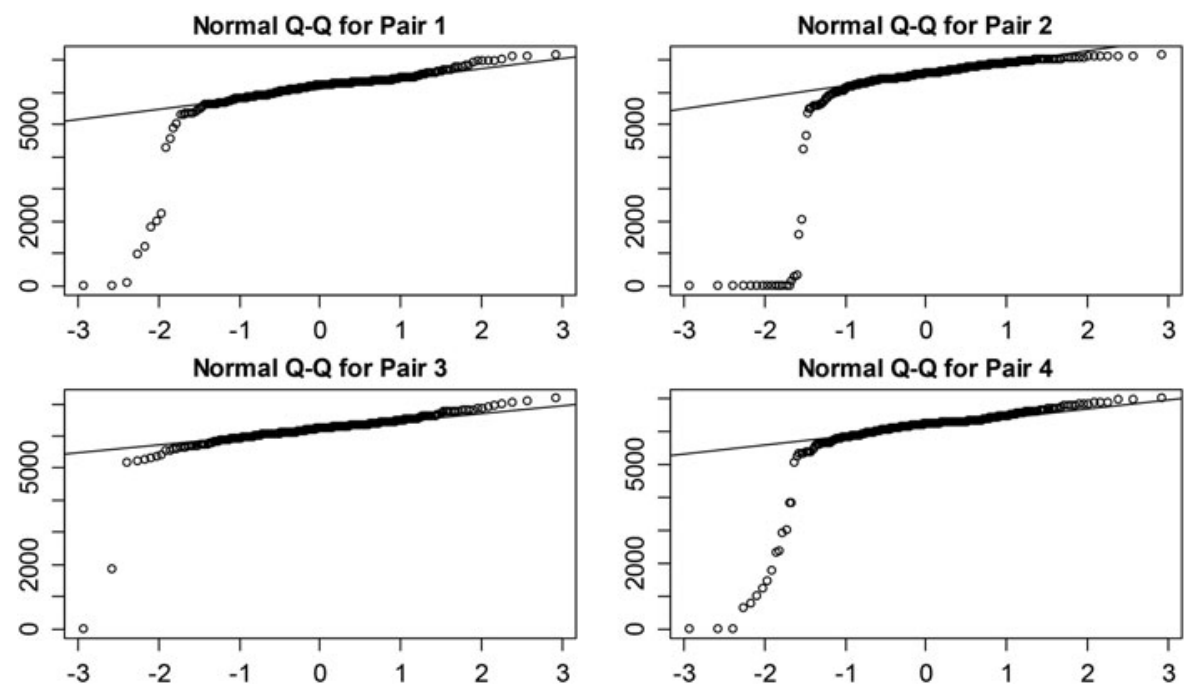

Figure 7. Normal quartile-quartile plots of results from each pair of antennae. 


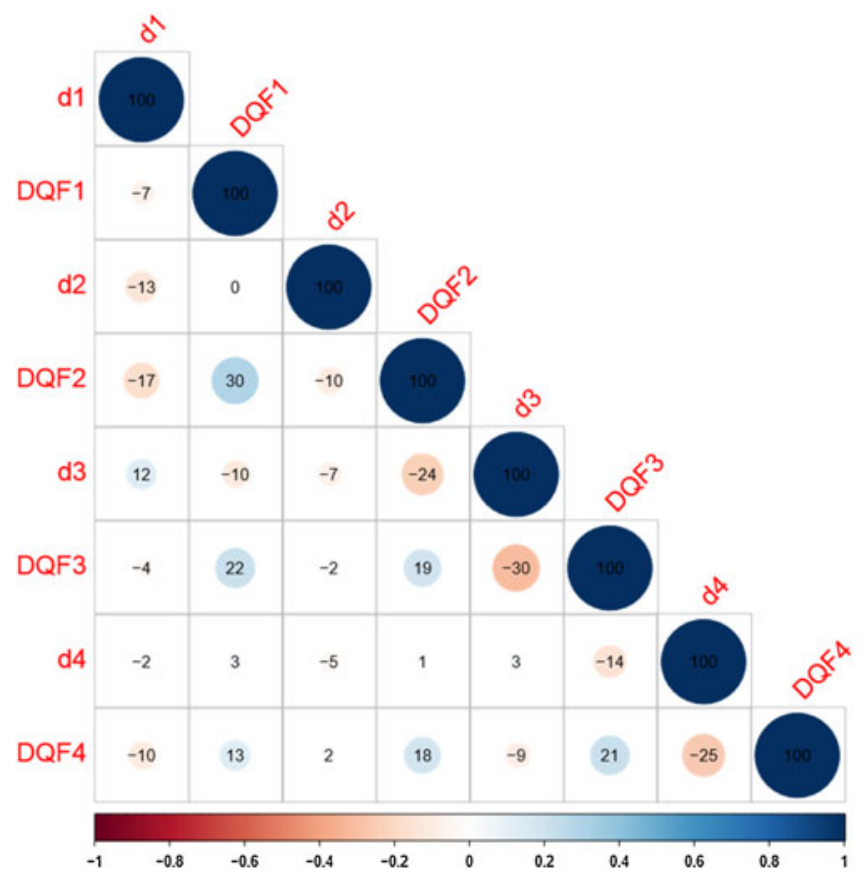

Figure 8. Plots of correlation between measurement results for antenna pairs.

with a normal distribution is presented in a normal quartile-quartile plot in Figure 7. Each point on the plot corresponds to one of the quartiles of the distribution of measurement results (y-coordinate) plotted against the same quartile of the normal distribution (x-coordinate). A straight line depicts the normal distribution. The distributions of the measurement results for pairs 3 and 4 are much closer to normal distribution than the distribution of measurement for pairs 1 and 2 .

An analysis of Figure 4 shows that in some epochs, the distance closest to the reference distance does not always originate from pair 3 or 4 .

Figure 8 depicts the correlation of DQFs and the distances between each pair of antennae. It is a graphical display of a correlation matrix. The size and colour of the circles depicts a correlation between the parameters on the intersection of each row and column. The numbers in the circles are values of correlation translated to percentages. The fact that there is no significant correlation between these variables suggests that the results from each pair can be treated independently.

Preliminary measurement results were processed using simple statistical parameters such as the average of distance and DQF, median of distance and DQF, minimum of distance and DQF, minimum of distance and DQF considering variance, maximum of distance and DQF (for each ranging cycle). The results are summarised in Table 2.

The total number of measured distances is 6000 ( 5 baselines $\times 4$ pairs $\times 300$ measurements). The mean residual calculated from all observations is $\widehat{d}_{t}=126 \mathrm{~cm}$, with a standard deviation $\sigma_{t}=569 \mathrm{~cm}$. The boundary of $\sigma_{t}\left( \pm \frac{1}{2} \sigma_{t}= \pm 284.5 \mathrm{~cm}\right)$ will be used in the rest of the paper as the maximum error boundary. 
Table 2. Statistics of the filtering results.

\begin{tabular}{|c|c|c|c|c|c|c|}
\hline Filtering method & $\operatorname{Min}[\mathrm{cm}]$ & $\operatorname{Median}[\mathrm{cm}]$ & Mean $[\mathrm{cm}]$ & $\operatorname{Max}[\mathrm{cm}]$ & $\sigma[\mathrm{cm}]$ & $\delta[\%]$ \\
\hline \multicolumn{7}{|c|}{$486 \mathrm{~cm}$} \\
\hline Average & 430 & 484 & 484 & 531 & 14 & $0 \cdot 41$ \\
\hline Median & 440 & 491 & 491 & 538 & 14 & $1 \cdot 02$ \\
\hline Minimum & 266 & 458 & 451 & 500 & 30 & $7 \cdot 20$ \\
\hline Minimum variance & 489 & 483 & 586 & 586 & 29 & $20 \cdot 58$ \\
\hline Maximum & 407 & 508 & 510 & 518 & 15 & $4 \cdot 94$ \\
\hline \multicolumn{7}{|c|}{$1276 \mathrm{~cm}$} \\
\hline Average & 600 & 1733 & 1834 & 3746 & 559 & $43 \cdot 73$ \\
\hline Median & 1089 & 1371 & 1370 & 1770 & 91 & $7 \cdot 37$ \\
\hline Minimum & 0 & 1219 & 936 & 1461 & 526 & $26 \cdot 65$ \\
\hline Minimum variance & 991 & 1328 & 1337 & 5330 & 255 & $4 \cdot 78$ \\
\hline Maximum & 1256 & 2912 & 3347 & 8041 & 1724 & $162 \cdot 30$ \\
\hline \multicolumn{7}{|c|}{$2000 \mathrm{~cm}$} \\
\hline Average & 2097 & 2317 & 2312 & 2476 & 78 & $15 \cdot 60$ \\
\hline Median & 2020 & 2378 & 2361 & 2613 & 128 & $18 \cdot 05$ \\
\hline Minimum & 1560 & 2083 & 2074 & 2409 & 144 & $3 \cdot 70$ \\
\hline Minimum variance & 1560 & 2332 & 2312 & 2764 & 202 & $15 \cdot 60$ \\
\hline Maximum & 2200 & 2521 & 2509 & 2764 & 97 & $25 \cdot 45$ \\
\hline \multicolumn{7}{|c|}{$4380 \mathrm{~cm}$} \\
\hline Average & 2425 & 4332 & 4266 & 5376 & 450 & $2 \cdot 60$ \\
\hline Median & 3372 & 4382 & 4361 & 4628 & 155 & 0.43 \\
\hline Minimum & 0 & 3884 & 3438 & 4560 & 1103 & $21 \cdot 51$ \\
\hline Minimum variance & 0 & 4391 & 4267 & 7123 & 877 & $2 \cdot 58$ \\
\hline Maximum & 4262 & 4657 & 4951 & 7123 & 664 & $13 \cdot 04$ \\
\hline \multicolumn{7}{|c|}{$6200 \mathrm{~cm}$} \\
\hline Average & 3983 & 6250 & 6078 & 6794 & 521 & 1.97 \\
\hline Median & 5245 & 6230 & 6193 & 7011 & 234 & $0 \cdot 11$ \\
\hline Minimum & 0 & 5887 & 5170 & 6469 & 1836 & $16 \cdot 61$ \\
\hline Minimum variance & 0 & 6268 & 6078 & 7149 & 1104 & $1 \cdot 97$ \\
\hline Maximum & 5956 & 6665 & 6673 & 7149 & 241 & $7 \cdot 63$ \\
\hline
\end{tabular}

Taking into account the lack of correlation it can be assumed that there are four independent data streams (one for each antenna pair). On the basis of the geometry, the most credible data stream is the one with the minimum measured distance value (it is most likely to reflect the direct path between two antennas without multipath effect). The credibility of the data obtained from a single pair of antennas is described by its DQF value.

In order to improve the final ranging results, on the basis of above assumptions, the following algorithm is proposed:

(A) Creating an intermediate data stream of consecutive $\mathrm{n}$ cycles

(1) read the data sample from each of the four pairs of antennas (in every ranging cycle)

(2) selection of the smallest value as most credible (without multipath effect) 


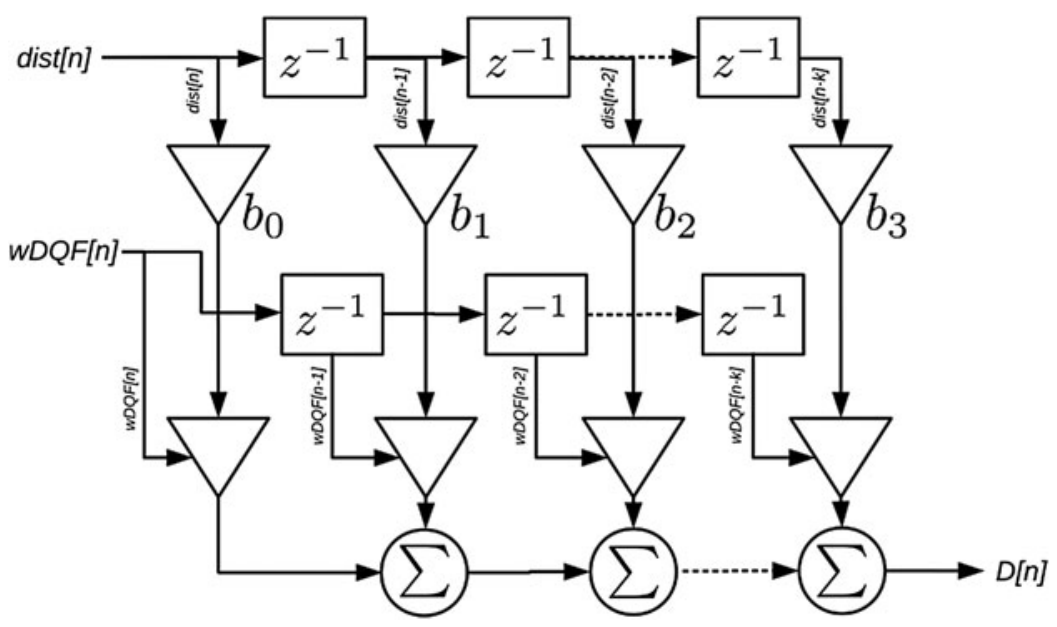

Figure 9. Adaptive moving average/FIR.

(3) add the value selected in point (2) to the intermediate stream

Denoting $a_{i}, b_{i}, c_{i}, d_{i}$ as values of distance measured in $\mathrm{i}^{\text {th }}$ cycle for pair $1,2,3$ and 4 respectively, from the geometric point of view the most credible distance $x_{i}$ is:

$$
x_{i}=\min \left\{a_{i}, b_{i}, c_{i}, d_{i}\right\}
$$

(B) Smoothing of the intermediate stream from point (A) using a moving average with a structure similar to the finite impulse response filter (FIR) is presented in Figure 9. In Figure 9, the notation $z^{-1}$ represents a previous sample.

According to this algorithm, the final value of distance $d$ is obtained by a sum of the last $k$ elements of an intermediate stream multiplied by the corresponding weights wDQF expressed as:

$$
w D Q F_{i}=\frac{D Q F_{i} \cdot 100}{\sum D Q F}
$$

where $\sum D Q F$ is a sum of DQF values in one cycle. Multiplication by 100 in the nominator is caused by the DQF values originally expressed as percentages. Additional smoothing effect, especially useful in a dynamic application, is obtained by weighting the last $n$ samples with the arbitrary selected constant weights $b_{0}, b_{1}, \ldots, b_{k}$. The choice of these parameters depends on the scenario in which the device will be used. These parameters provide a smoothing of movement, so they will depend on the system dynamics. In the example presented in this paper these values decrease in arithmetical progression. The algorithm can be presented in the form of Equation (5).

$$
d_{n}=\sum_{k=0}^{k=N} x_{(n-k)} w D Q F_{(n-k)} b_{k}
$$




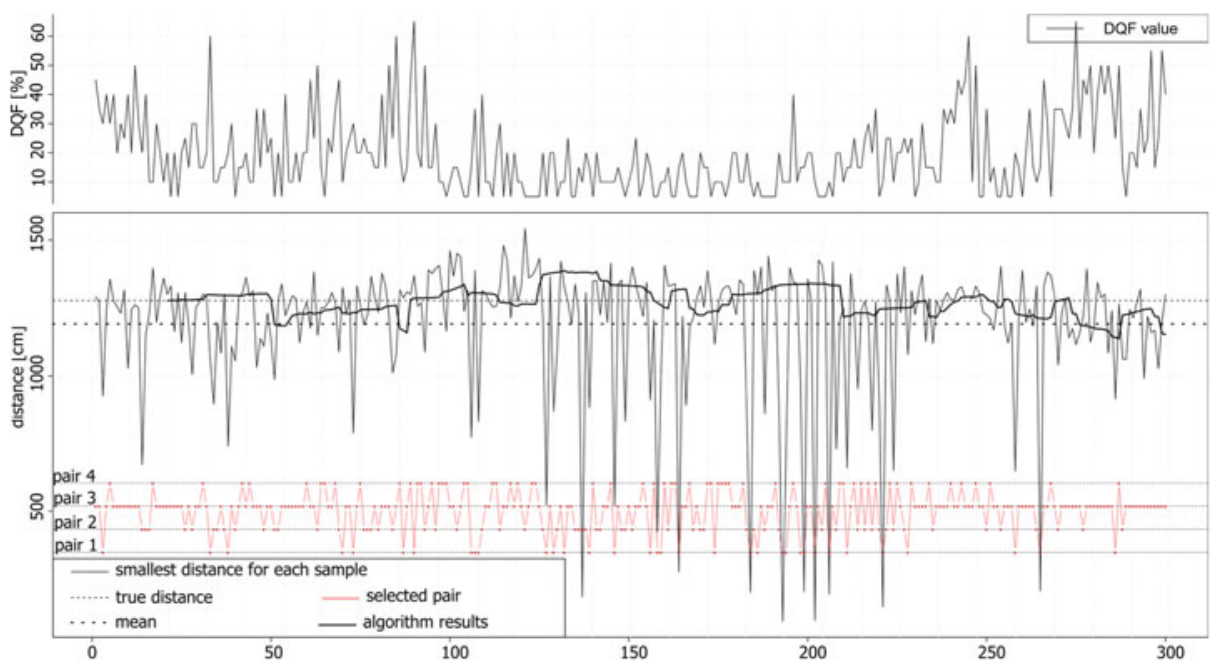

Figure 10. Minimum distances for each sample.

Table 3. Number of selections for each pair.

\begin{tabular}{lllll}
\hline Pair & 1 & 2 & 3 & 4 \\
\hline No. selections & 34 & 60 & 146 & 60 \\
\hline
\end{tabular}

The results of applying of the algorithm presented above (with FIR length $\mathrm{N}=20$ ) are presented in Figure 10 along with corresponding DQF values and selected pairs of antennas. The number of selections for each pair of antennas is summarised in Table 3. In almost $50 \%$ of cycles pair number 3 was selected. This suggests that for antenna pair 3 the distance is measured in a way closest to the LOS.

The bold line in Figure 10 depicts the results of computations. It is much smoother than the raw measurements. Statistics of the results are provided in Table 4.

The results of all of the filtration methods presented above are summarised in Figure 11. Horizontal bars represent the mean residuals for each method at each distance. Black error bars represent standard deviations.

For each baseline the mean results of proposed filtering method are inside the $1 \sigma$ boundary. For the presented method the mean residual is increasing with growing distance. In the case of the rest of the methods presented in this paper there is no dependency between distance and mean residual. The smallest residuals, comparable for each filtering method are for the shortest distance. This is because of a short distance - the DQF value is $100 \%$ for almost all measurements, so weights are equal and the algorithm is simpler to average.

5. CONCLUSIONS. In this paper, the results of distance measurements performed with AT86RF233 are presented and analysed. The measurement experiment was prepared using an REB233SMADEK device. For the test purposes 6000 data samples were collected. Performed tests confirmed the usability of AT86RF233 in ranging. It provides better ranging accuracy then TOF or RSSI methods. 
Table 4. Statistics of the filtering results $[\mathrm{cm}]$.

\begin{tabular}{lcccccc}
\hline Distance $[\mathrm{cm}]$ & Min $[\mathrm{cm}]$ & Median[cm] & Mean[cm] & Max $[\mathrm{cm}]$ & $\sigma[\mathrm{cm}]$ & $\delta[\%]$ \\
\hline 486 & 407 & 459 & 467 & 480 & 12 & $3 \cdot 10$ \\
1276 & 1148 & 1261 & 1265 & 1380 & 46 & $0 \cdot 86$ \\
2000 & 1889 & 2036 & 2062 & 2279 & 95 & $3 \cdot 91$ \\
4380 & 663 & 4355 & 4410 & 4430 & 700 & $0 \cdot 68$ \\
6200 & 5264 & 5857 & 5925 & 6019 & 218 & $2 \cdot 91$ \\
\hline
\end{tabular}

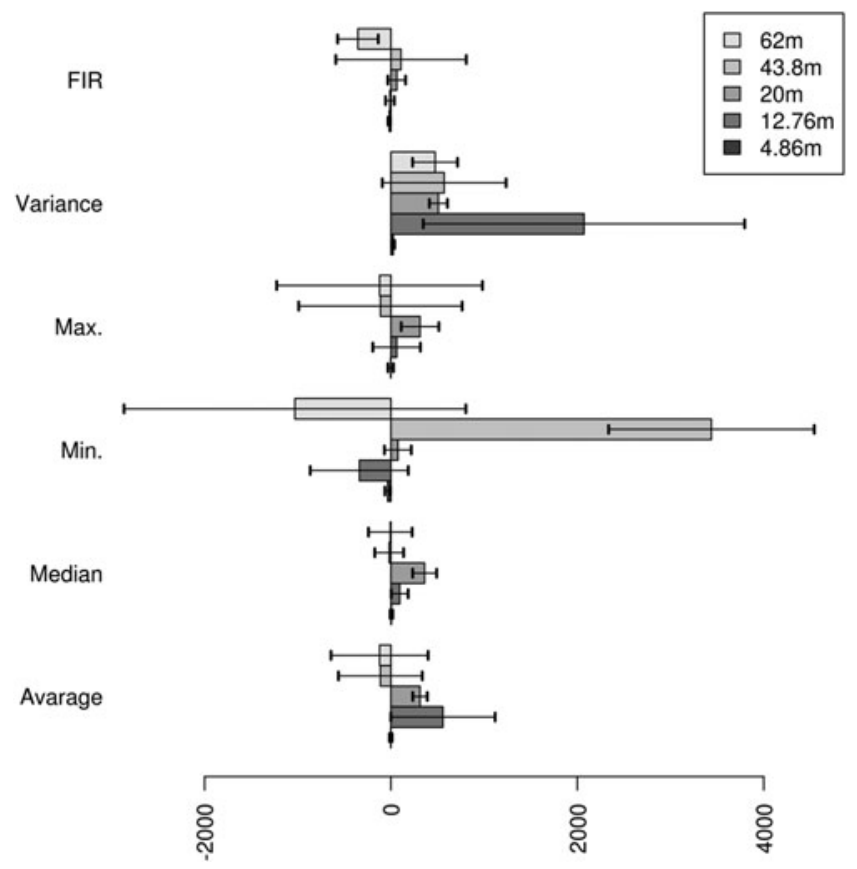

Figure 11. Mean residuals with error bars for each filtering method at different distances.

An algorithm for data processing was proposed based on preliminary statistical evaluation. It consists of a sample selection (forming an intermediate stream) stage and smoothing of the results stage. In the second stage, a weighted moving average is used.

The obtained results show that raw measurements are difficult to interpret and apply in ranging. A simple way to use this data is to apply simple statistical tools such as mean, median or variance (Table 2). A major improvement in ranging results can be obtained using the proposed algorithm. In the case of measurement at $4.86 \mathrm{~m}$ distance, the DQF values are saturated so the weighting procedure did not improve the results significantly. The improvement is visible at longer distances where the DQF values varies. For example for the $12.76 \mathrm{~m}$ value, using simple statistics gave the best standard deviation of $91 \mathrm{~cm}$ while standard deviation after applying the proposed algorithm is $46 \mathrm{~cm}$. The mean residual value also improved from $61 \mathrm{~cm} \mathrm{(4.78 \%} \mathrm{of} \mathrm{total} \mathrm{distance)} \mathrm{to}$ $11 \mathrm{~cm}(0 \cdot 86 \%$ of total distance).

The obtained results encourage the use of ranging based on phase shift measurements and the ZigBee protocol in positioning applications. The combination of 
network communication and ranging in one device allows for further work on collaborative navigation, for example, in indoor applications.

\section{REFERENCES}

Atmel. (2013). Atmel AVR2150: RTB Evaluation Application - User's Guide, First edition.

Casilari, C., Cano-García, J.M. and Campos-Garrido, G. (2010). Modelling of current consumption in 802·15·4/Zigbee sensor motes. Sensors, 10(6), 5443-5468.

Chen, Q., H. Liu, H., Yu, M. and Guo, H. (2012). RSSI ranging model and 3D indoor positioning with Zigbee network. Position Location and Navigation Symposium (PLANS) IEEE/ION, 1233-1239.

Günther, A. and Hoene, C. (2005). Measuring round trip times to determine the distance between wlan nodes. In Boutaba, R., Almeroth, K., Puigjaner, R, Shen, S. and Black, J.P., editors, NETWORKING 2005. Networking Technologies, Services, and Protocols; Performance of Computer and Communication Networks; Mobile and Wireless Communications Systems, volume 3462 of Lecture Notes in Computer Science, 768-779. Springer Berlin Heidelberg, 2005.

Indelman, V., Gurfil, P., Rivlin, E. and Rotstein, H. (2011). Distributed vision-aided cooperative localization and navigation based on three-view geometry. Aerospace Conference, IEEE, 1-20.

Hui Liu, H. Darabi, Banerjee, P. and Liu, J. (2007). Survey of wireless indoor positioning techniques and systems. Systems, Man, and Cybernetics, Part C: Applications and Reviews, IEEE Transactions on, 37 (6), 1067-1080.

Nilsson, J.-O., Zachariah, D., Skog, I. and Händel, P. (2013). Cooperative localization by dual foot-mounted inertial sensors and inter-agent ranging. EURASIP Journal on Advances in Signal Processing 2013, 2013: 164 CoRR, abs/1304·3663. (http://arxiv.org/pdf/1304.3663v4.pdf)

Obst, M., Shubert, R., Mattern, N., Liberto, C., Romon, S. and Khoudour, L. (2012). Cooperative Gnss Localization in Urban Environments - Results from rhe Covel Project. Proceedings of the 19th ITS World Congress, Vienna, Austria, 1-13.

Smieja, M. (2014). The use of RF communication devices in ranging applications. Selected papers from the 9th International Conference Environmental Engineering Vilnius, 22-23 May 2014.

Wu, L., Meng, M.Q.-H, Lin, Z., He, W., Peng, C. and Liang, H. (2009). A practical evaluation of radio signal strength for mobile robot localization. Robotics and Biomimetics (ROBIO), 2009 IEEE International Conference on, 516-522. 